\title{
Performance Analysis of Triple Selection Diversity Over Exponentially Correlated Nakagami- $m$ Fading Channels
}

\author{
George K. Karagiannidis, Member, IEEE, Dimitris A. Zogas, Student Member, IEEE, and Stavros A. Kotsopoulos
}

\begin{abstract}
In this letter, an approach to the performance analysis of a triple selection-diversity system over exponentially correlated Nakagami- $m$ fading channels is presented. Closed-form expressions of converged sums for both outage and average error probabilities are derived. Numerical results are presented to point out the effect of the fading correlation, the fading severity, as well as the improvement achieved by the triple selection combining compared with the corresponding dual diversity case.
\end{abstract}

Index Terms-Fading correlation, Nakagami- $m$ fading channels, Rayleigh fading channels, selection diversity.

\section{INTRODUCTION}

D IVERSITY reception using multiple antennas has been successfully used in wireless communications systems to mitigate the effect of channel fading and to improve the received signal-to-noise ratio (SNR). Among several types of diversity combining, selection combining (SC) is the least complicated since the processing is performed only on one of the diversity branches. Traditionally, in SC, the branch with the highest SNR is selected, which corresponds to the strongest signal, if equal noise power is assumed among the branches. However, sometimes it is better to analyze the SC performance using the voltage SNR, defined as the square root of the power SNR [1]. The performance analysis of SC, assuming independent channel fading, has been studied extensively in the literature [2]. However, independent fading assumes antenna elements be placed sufficiently far apart, which is not always realized in practice due to insufficient spacing, especially in small-size terminals. In such cases, the fading among the channels is correlated, resulting in a degradation of the diversity gain obtained. Several correlation models have been proposed and used for the performance analysis of various wireless systems, corresponding to specific modulation, detection, and diversity combining schemes. One of the most frequently used models is the exponential correlation model. This model corresponds to the scenario of multichannel reception from equispaced diversity antennas, in which the correlation between the pairs of combined signals decays as the spacing between the antennas increases [2]. In the literature, there are several approaches to the performance analysis of SC in the presence of correlative Nakagami- $m$ or Rayleigh fading

Paper approved by G. M. Vitetta, the Editor for Equalization and Fading Channels of the IEEE Communications Society. Manuscript received June 14, 2002; revised October 2, 2002; October 11, 2002; and January 16, 2003.

G. K. Karagiannidis is with the Institute for Space Applications and Remote Sensing, National Observatory of Athens, 15236 Athens, Greece (e-mail: gkarag@space.noa.gr).

D. A. Zogas and S. A. Kotsopoulos are with the Electrical and Computer Engineering Department, University of Patras, 26444 Patras, Greece (e-mail: zogas@space.noa.gr; kotsop@ee.upatras.gr).

Digital Object Identifier 10.1109/TCOMM.2003.815070 channels, but they are limited to the case of dual diversity [1], [3], [4]. As far as higher order diversity is concerned, Ugweje and Aalo in [5], using a previously published formula for the multivariate gamma probability density function (PDF), derived expressions for the $n$-branches SC output PDF and cumulative distribution function (CDF) in the form of multiple integral of infinite sum of Laguerre polynomials. In [5] the dual SC case is analyzed, due to the complexity of the proposed formulation. Finally, Zhang and Lu in [6] studied the performance of multibranch SC over correlative fading channels via the joint characteristic functions of the inputs SNRs, but the derived formula for the SC output PDF is in the form of a multifold integral with infinite limits.

In this letter, using closed-form expressions for the multivariate Nakagami- $m$ PDF and CDF, recently proposed by the authors in [7], a unified performance analysis of a triple SC over exponentially correlated Nakagami- $m$ fading channels is presented. Both outage and average error performance are investigated. The effect of the fading correlation and the fading severity on the performance of the SC is also presented. The proposed approach in this paper can be efficiently extended to the $N$ branches SC, using [7] and similar mathematical analysis.

\section{OUtage Probability}

In the last years, there has been a continuing interest in modeling various propagation channels with the Nakagami- $m$ model, which describes multipath scattering with relatively large delay-time spreads, with different clusters of reflected waves. If $r$ is a Nakagami- $m$ variable, then its corresponding PDF is described by [2]

$$
f_{r}(r)=\frac{2 r^{2 m-1}}{\Gamma(m) \Omega^{m}} \exp \left(-\frac{r^{2}}{\Omega}\right), \quad r \geq 0
$$

where $\Gamma(\cdot)$ is the Gamma function, $\Omega=\overline{r^{2}} / m$, with $\overline{r^{2}}$ being the average signal power, and $m$ is the inverse normalized variance of $r^{2}$ which must satisfy $m \geq 1 / 2$, describing the fading severity. Recently, the authors in [7] proposed a useful formulation for the multivariate (joint) Nakagami- $m$ PDF and CDF with an exponential correlation matrix that can be efficiently applied in the performance analysis of the triple SC. According to this formulation, the trivariate Nakagami- $m$ PDF can be written as

$$
\begin{aligned}
f_{r_{1}, r_{2}, r_{3}}\left(r_{1}, r_{2}, r_{3}\right)= & \frac{r_{1}^{m} r_{2} r_{3}^{m} \exp \left(-\frac{r_{1}^{2}+\left(\rho^{2}+1\right) r_{2}^{2}+r_{3}^{2}}{2\left(1-\rho^{2}\right)}\right)}{2^{m-1} \Gamma(m) \rho^{2(m-1)}\left(1-\rho^{2}\right)^{2}} \\
& \times I_{m-1}\left(\frac{\rho}{1-\rho^{2}} r_{1} r_{2}\right) I_{m-1}\left(\frac{\rho}{1-\rho^{2}} r_{2} r_{3}\right)
\end{aligned}
$$


whereas $I_{v}(\cdot)$ is the first kind and $v$ th-order modified Bessel function and $\rho$ is the power correlation coefficient defined as $\operatorname{cov}\left(r_{i}^{2}, r_{j}^{2}\right) / \sqrt{\operatorname{var}\left(r_{i}^{2}\right) \operatorname{var}\left(r_{j}^{2}\right)}$ with $0 \leq \rho<1$. In (2) is assumed, without loss of generality, and for simplification purposes of the correlation matrix of $r_{1}, r_{2}, r_{3}, \Omega_{i}=2 \sigma_{i}^{2}, i=1,2$, 3 , and the variance $\sigma_{i}^{2}=1$. The corresponding trivariate $\mathrm{CDF}$ is extracted in [7] as

$$
\begin{aligned}
F_{R_{1}, R_{2}, R_{3}}( & \left.R_{1}, R_{2}, R_{3}\right) \\
= & \frac{\left(1-\rho^{2}\right)^{m}}{\Gamma(m)} \sum_{i_{1}, i_{2}=0}^{\infty} \frac{\rho^{2 i_{1}+2 i_{2}}}{\left(\rho^{2}+1\right)^{i_{1}+i_{2}+m}} \\
& \times \frac{\gamma\left(i_{1}+m, \frac{R_{1}^{2}}{2\left(1-\rho^{2}\right)}\right) \gamma\left(i_{1}+i_{2}+m, \frac{\left(\rho^{2}+1\right) R_{2}^{2}}{2\left(1-\rho^{2}\right)}\right)}{i_{1} ! i_{2} ! \Gamma\left(i_{1}+m\right) \Gamma\left(i_{2}+m\right)} \\
& \times \gamma\left(i_{2}+m, \frac{R_{3}^{2}}{2\left(1-\rho^{2}\right)}\right)
\end{aligned}
$$

with $\gamma(\cdot)$ being the "lower" incomplete Gamma function [8, (6.5.2)]. The nested double sum in (3) converges rapidly and a few terms need to be summed in order to obtain accuracy at the seventh significant digit. Also, in [7] a formula for the bound of the error resulting after the truncation of the nested infinite series in (3) is presented. Defining now the instantaneous SNR per symbol and per channel $\zeta_{i}=r_{i}^{2} E_{s} / N_{0}, i=1,2,3$, with $E_{s} / N_{0}$ being the symbol energy-to-Gaussian noise spectral density ratio, and the average SNR per symbol $\bar{\zeta}=\overline{\zeta_{i}}=$ $\overline{r_{i}^{2}} E_{s} / N_{0}=2 m E_{s} / N_{0}$, the joint trivariate $\mathrm{CDF}$ of $\zeta_{1}, \zeta_{2}, \zeta_{3}$ can be found directly from (3) as

$$
\begin{aligned}
& F_{\zeta_{1}, \zeta_{2}, \zeta_{3}}\left(\zeta_{1}, \zeta_{2}, \zeta_{3}\right)= \\
& \quad h f i l l F_{R_{1}, R_{2}, R_{3}}\left(\sqrt{\frac{2 m \zeta_{1}}{\bar{\zeta}}}, \sqrt{\frac{2 m \zeta_{2}}{\bar{\zeta}}}, \sqrt{\frac{2 m \zeta_{3}}{\bar{\zeta}}}\right) .
\end{aligned}
$$

The assumption of identical powers in all three branches is reasonable if the diversity channels are closely spaced and the gain of each channel is such that all the noise powers are equals [2]. Using (4), the outage probability of the SC output SNR, $P_{\text {out }}$, defined as the probability that all $\zeta_{1}, \zeta_{2}$, and $\zeta_{3}$ fall below a threshold $\zeta_{t h}$, can be evaluated as

$$
P_{\text {out }}=F_{\zeta}\left(\zeta_{t h}\right)=F_{\zeta_{1}, \zeta_{2}, \zeta_{3}}\left(\zeta_{t h}, \zeta_{t h}, \zeta_{t h}\right)
$$

\section{Average Error Performance}

The PDF of the triple SC output SNR, $f_{\zeta}(\zeta)$, can be derived as

$$
\begin{aligned}
f_{\zeta}(\zeta)= & \frac{d F_{\zeta}(\zeta)}{d \zeta} \\
= & \frac{\left(1-\rho^{2}\right)^{m}}{\Gamma(m)} \sum_{i_{1}, i_{2}=0}^{\infty} \\
& \times \frac{\rho^{2 i_{1}+2 i_{2}}\left[G_{1}+G_{2}+G_{3}\right]}{\left(\rho^{2}+1\right)^{i_{1}+i_{2}+m} i_{1} ! i_{2} ! \Gamma\left(m+i_{1}\right) \Gamma\left(m+i_{2}\right)}
\end{aligned}
$$

where

$$
\begin{aligned}
G_{1} & =a_{1}^{b_{1}} \zeta^{b_{1}-1} e^{-a_{1} \zeta} \gamma\left(b_{2}, a_{2} \zeta\right) \gamma\left(b_{3}, a_{1} \zeta\right) \\
G_{2} & =a_{2}^{b_{2}} \zeta^{b_{2}-1} e^{-a_{2} \zeta} \gamma\left(b_{1}, a_{1} \zeta\right) \gamma\left(b_{3}, a_{1} \zeta\right) \\
G_{3} & =a_{1}^{b_{3}} \zeta^{b_{3}-1} e^{-a_{1} \zeta} \gamma\left(b_{1}, a_{1} \zeta\right) \gamma\left(b_{2}, a_{2} \zeta\right) \\
a_{1} & =\frac{m}{\left(1-\rho^{2}\right) \bar{\zeta}}, a_{2}=\frac{\left(\rho^{2}+1\right) m}{\left(1-\rho^{2}\right) \bar{\zeta}} \\
b_{1} & =m+i_{1}, b_{2}=m+i_{1}+i_{2}, b_{3}=m+i_{2} .
\end{aligned}
$$

The average bit-error rate (ABER), $\overline{P_{e}}$, can be extracted averaging the conditional error probability, $P(\varepsilon / \zeta)$, over the PDF of the output SNR. For binary differentially phase-shift keying (BDPSK) and noncoherent binary frequency-shift keying (BFSK), $\overline{P_{e}}$, can be written as [2]

$$
\overline{P_{e}}=\int_{0}^{\infty} \frac{1}{2} e^{-g \zeta} f_{\zeta}(\zeta) d \zeta
$$

with $g=1$ for BDPSK and $g=1 / 2$ for BFSK. As the quantity in the double sum of (6) is Riemann integrable and converges uniformly on the range $[0, \infty)$, the order of integration and summation is interchangeable. The integrals produced in (7) have the following form:

$$
\int_{0}^{\infty} e^{-a x} x^{b} \gamma\left(d_{1}, c_{1} x\right) \gamma\left(d_{2}, c_{2} x\right) d x
$$

which, using $[8,(6.5 .12)]$ and applying the Kummer transformation $[9,(9.20)]$, can be written as

$$
\begin{aligned}
\int_{0}^{\infty} e^{-a x} x^{b} & \gamma\left(d_{1}, c_{1} x\right) \gamma\left(d_{2}, c_{2} x\right) \\
= & \frac{c_{1}^{d_{1}} c_{2}^{d_{2}}}{d_{1} d_{2}} \int_{0}^{\infty} \frac{x^{d_{1}+d_{2}+b}}{e^{\left(a+c_{1}+c_{2}\right)}} \\
& \times{ }_{1} F_{1}\left(1, d_{1}+1 ; c_{1} x\right){ }_{1} F_{1}\left(1, d_{2}+1 ; c_{2} x\right) d x
\end{aligned}
$$

where ${ }_{1} F_{1}\left(z_{1}, z_{2}, x\right)$ is the well-known confluent hypergeometric function. The integral in the second part of (9) can be written in a closed form using [10, App. C] resulting in

$$
\begin{aligned}
\int_{0}^{\infty} e^{-a x} x^{b} \gamma\left(d_{1}, c_{1} x\right) \gamma\left(d_{2}, c_{2} x\right) \\
=\frac{c_{1}^{d_{1}} c_{2}^{d_{2}} \Gamma\left(b+d_{1}+d_{2}+1\right)}{d_{1} d_{2}\left(a+c_{1}+c_{2}\right)^{\left(b+d_{1}+d_{2}+1\right)}} \\
\times F_{2}\left(b+d_{1}+d_{2}+1 ; 1,1 ; d_{1}+1, d_{2}+1\right) \\
\left.; \frac{c_{1}}{a+c_{1}+c_{2}}, \frac{c_{2}}{a+c_{1}+c_{2}}\right)
\end{aligned}
$$

with $F_{2}$ being the hypergeometric function of two variables defined in [9, (9.180/2)]. Using (10), and after some algebraic manipulations, the ABER for BDPSK and BFSK can be written as shown in (11) at the bottom of the next page. 


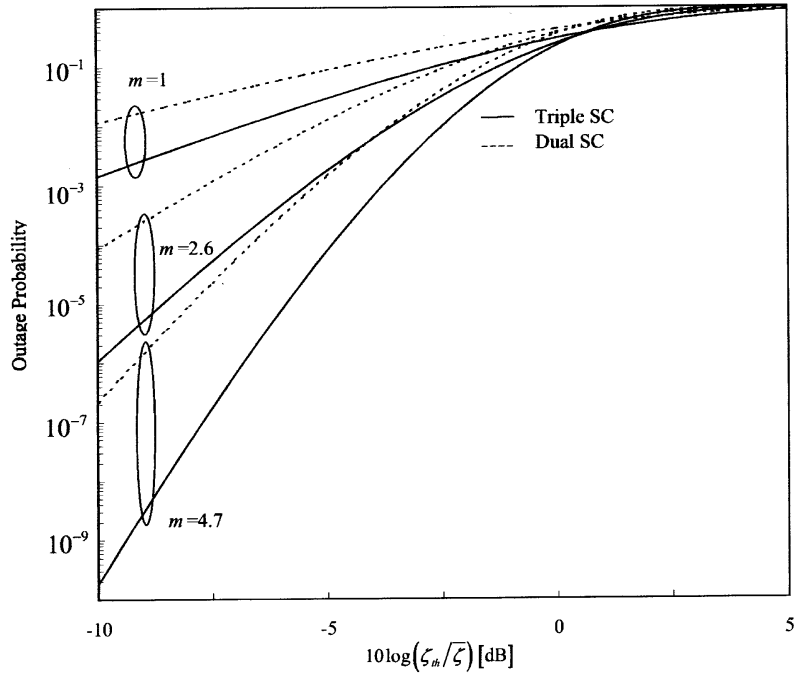

Fig. 1. Outage probability, $P_{\text {out }}$, versus $\zeta_{t h} / \bar{\zeta}$, for dual and triple SC, $\rho=$ 0.5 , and several values of $m$.

Now, working as in [3], it can be easily recognized that there is a similarity between the formulae of $P(\varepsilon / \zeta)$ for several modulation schemes. Hence, single finite-interval integration suffices for direct computation of the desired ABER. For example, in coherent BPSK or orthogonal BFSK, $\overline{P_{e}}$ can be derived as $\overline{P_{e}}=(1 / \pi) \int_{0}^{\pi / 2} \overline{P_{e}}(\theta) d \theta$, where $\overline{P_{e}}(\theta)$ is given in (11) with $g$ to be replaced with $g(\theta)=\left(g /\left(\sin ^{2}(\theta)\right)\right)$ and $g=1, g=1 / 2$ for BPSK and orthogonal BFSK, respectively.

\section{NumericAl RESUlTS AND DisCUSSION}

The double infinite sum in (11) converges rapidly and a mean number of five terms (for each sum) is adequate to be summed for accuracy $10^{-10}$ at $\bar{\zeta}=10 \mathrm{~dB}$, and three terms for accuracy $10^{-15}$ at $\bar{\zeta}=20 \mathrm{~dB}$. In Fig. 1, the outage probability is plotted versus the normalized threshold $\zeta_{t h} / \bar{\zeta}$ for the dual and triple SC, $\rho=0.5$ and for several values of $m$. It is evident that the

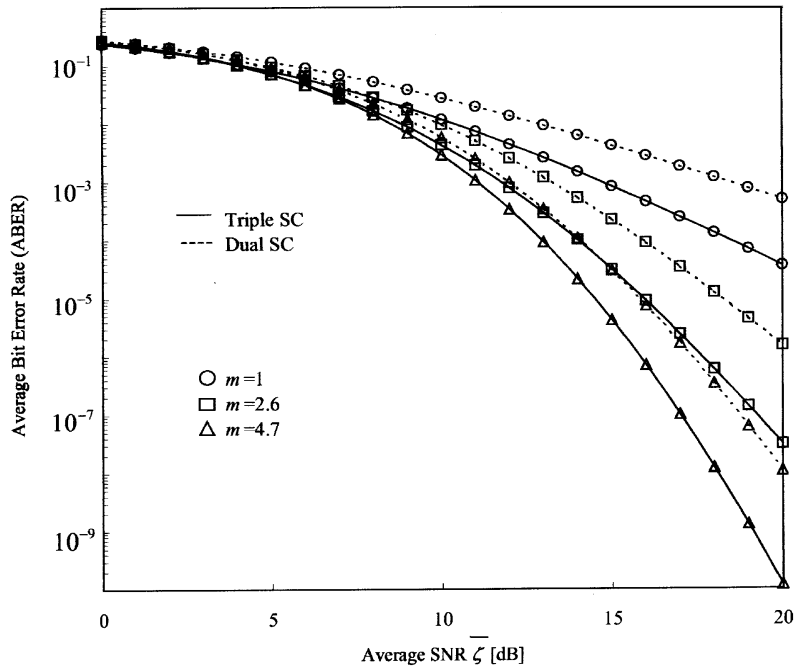

Fig. 2. ABER, $\overline{P_{e}}$, versus $\bar{\zeta}$, for dual and triple SC in noncoherent BFSK, $\rho=0.5$, and several values of $m$.

outage performance degrades as the fading severity increases ( $m$ decreases) as well as the improvement offered by the triple SC, compared to the dual one, is lower for high fading severity environments (small values of $m$ ). At $P_{\text {out }}=0.1$ and $m=1$ (Rayleigh), the improvement offered by the triple SC is about $2.5 \mathrm{~dB}$. For $P_{\text {out }}=10^{-3}$, the corresponding improvement is about $2.5 \mathrm{~dB}$ for $m=2.6$ and $1.25 \mathrm{~dB}$ for $m=4.7$. Fig. 2 plots the ABER for noncoherent BFSK, for both dual and triple SC, $\rho=0.5$, and several values of $m$. It is evident that the triple diversity offers an improvement to the error performance. When $\overline{P_{e}}=10^{-3}$ the gain offered by the triple SC compared with the dual-diversity case is about $3.5 \mathrm{~dB}$ for $m=1$, and when $\overline{P_{e}}=10^{-4}$ the corresponding gain is $2 \mathrm{~dB}$ for $m=2.6$ and 1 $\mathrm{dB}$ for $m=4$.7. Finally, Figs. 3 and 4 show the impact of the fading correlation to the outage probability (for $\zeta_{t h} / \bar{\zeta}=0 \mathrm{~dB}$ ) and average error performance (coherent BPSK with $\bar{\zeta}=15$ $\mathrm{dB}$ ) correspondingly, for several values of $m$. As expected, an

$$
\bar{P}_{e}=\frac{1}{2 \Gamma(m)} \sum_{i_{1}, i_{2}=0}^{\infty} \frac{m^{2 i_{1}+2 i_{2}+3 m} \rho^{2 i_{1}+2 i_{2}}\left(1-\rho^{2}\right)^{m}}{\left[\left(\rho^{2}+3\right) m+\left(1-\rho^{2}\right) \bar{\zeta} g\right]^{2 i_{1}+2 i_{2}+3 m}} \times \frac{\Gamma\left(2 i_{1}+2 i_{2}+3 m\right)\left[U_{1}+U_{2}+U_{3}\right]}{i_{1} ! i_{2} ! \Gamma\left(m+i_{1}\right) \Gamma\left(m+i_{2}\right)}
$$

with

$$
\begin{gathered}
U_{1}=\frac{F_{2}\left(3 m+2 i_{1}+2 i_{2}+1 ; 1,1 ; m+i_{1}+i_{2}+1, m+i_{2}+1 ; v_{2}, v_{1}\right)}{\left(m+i_{1}+i_{2}\right)\left(m+i_{2}\right)} \\
U_{2}=\frac{F_{2}\left(3 m+2 i_{1}+2 i_{2}+1 ; 1,1 ; m+i_{1}+1, m+i_{2}+1 ; v_{1}, v_{1}\right)}{\left(m+i_{1}\right)\left(m+i_{2}\right)} \\
U_{3}=\frac{F_{2}\left(3 m+2 i_{1}+2 i_{2}+1 ; 1,1 ; m+i_{1}+1, m+i_{1}+i_{2}+1 ; v_{1}, v_{2}\right)}{\left(m+i_{1}\right)\left(m+i_{1}+i_{2}\right)} \\
v_{1}=\frac{m}{\left(\rho^{2}+3\right) m+\left(1-\rho^{2}\right) \bar{\zeta} g} \\
v_{2}=\frac{m\left(\rho^{2}+1\right)}{\left(\rho^{2}+3\right) m+\left(1-\rho^{2}\right) \bar{\zeta} g}
\end{gathered}
$$




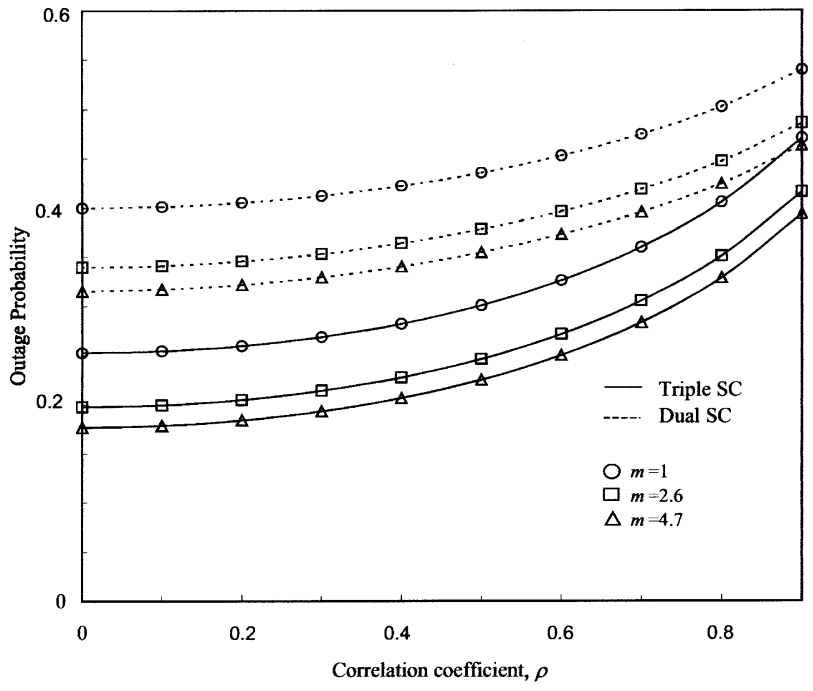

Fig. 3. Outage probability, $P_{\text {out }}$, versus the correlation coefficient $\rho$, for dual and triple $\mathrm{SC}, \zeta_{t h} / \bar{\zeta}=0 \mathrm{~dB}$, and several values of $m$.

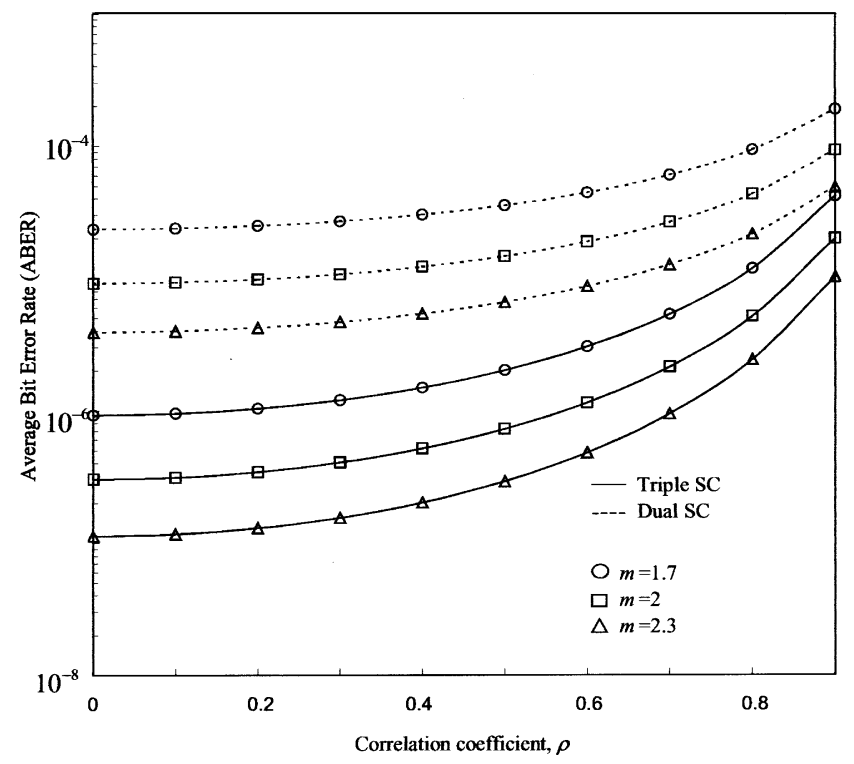

Fig. 4. ABER, $\overline{P_{e}}$, versus the correlation coefficient $\rho$ for dual and triple SC, $\bar{\zeta}=15 \mathrm{~dB}$, and several values of $m$.

increase of $\rho$ leads to an increase of the outage probability and the ABER, while the slope of the curves preserves regardless of the value of the $m$ parameter. Moreover, the triple-diversity case is more sensitive to the fading correlation effects. The curves for the dual selection-diversity case were obtained using the analysis proposed in [7] with $n=2$, and following the same procedure as in Sections II and III.

\section{CONCLUSIONS}

In this letter, a unified performance analysis for the triple SC over exponentially correlated Nakagami- $m$ fading channels is presented. Closed-form expressions of converged sums for the outage probability and ABER are derived. Numerical results show that the improvement obtained using a three-branch selection combiner is considerable compared to the dual diversity case. Moreover, the effect of the fading correlation to the system performance is more significant in the triple diversity case, compared with the dual one.

\section{ACKNOWLEDGMENT}

The authors would like to thank the anonymous reviewers and the Editor, G. Vitetta, for their valuable comments that considerably improved the quality of this letter.

\section{REFERENCES}

[1] K. Dietze, C. B. Dietrich, Jr., and W. L. Stutzman, "Analysis of a twobranch maximal ratio and selection diversity system with unequal SNRs and correlated inputs for a Rayleigh fading channel," IEEE Trans. Wireless Commun., vol. 1, pp. 274-281, Apr. 2002.

[2] M. K. Simon and M.-S. Alouini, Digital Communication Over Fading Channels, 1st ed. New York: Wiley, 2000.

[3] — , "A unified performance analysis of digital communications with dual selective combining diversity over correlated Rayleigh and Nakagami- $m$ fading channels," IEEE Trans. Commun., vol. 47, pp. 33-43, Jan. 1999.

[4] G. M. Vitetta, U. Mengali, and D. P. Taylor, "An error probability formula for noncoherent orthogonal binary FSK with dual diversity on correlated Rician channels," IEEE Commun. Lett., vol. 3, pp. 43-45, Feb. 1999.

[5] O. C. Ugweje and V. Aalo, "Performance of selection diversity system in correlated Nakagami fading," in Proc. IEEE VTC'97, Phoenix, AZ, May 1997, pp. 1488-1492.

[6] Q. T. Zhang and H. G. Lu, "A general analytical approach to multibranch selection combining over various spatially correlated fading channels," IEEE Trans. Commun., vol. 50, pp. 1066-1073, July 2002.

[7] G. K. Karagiannidis, D. A. Zogas, and S. A. Kotsopoulos, "On the multivariate Nakagami- $m$ distribution with exponential correlation," IEEE Trans. Commun., vol. 51, pp. 1240-1244, Aug. 2003.

[8] M. Abramovitz and I. A. Stegun, Handbook of Mathematical Functions With Formulas, Graphs, and Mathematical Tables, 9th ed. New York: Dover, 1972.

[9] I. S. Gradshteyn and I. M. Ryzhik, Table of Integrals, Series, and Products, 5th ed. New York: Academic, 1994.

[10] A. Annamalai, C. Tellambura, and V. K. Bhargava, "Equal-gain diversity receiver performance in wireless channels," IEEE Trans. Commun., vol. 48, pp. 1732-1745, Oct. 2000. 